\title{
Shape from Shading and Viscosity Solutions
}

\author{
Emmanuel Prados ${ }^{1}$, Olivier Faugeras ${ }^{1}$, and Elisabeth Rouy ${ }^{2}$ \\ 1 INRIA, 2004 route des Lucioles, BP 93, 06902 Sophia-Antipolis Cedex, France \\ emmanuel.prados (Olivier.Faugeras) @sophia.inria.fr \\ 2 Institut National des Sciences Appliquées de Lyon, MAPLY - UMR5585, \\ Laboratoire de Modélisation Mathématique et de Calcul Scientifique, Bâtiment 401, \\ 20 Avenue Albert Einstein, 69621 VILLEURBANNE Cedex, France \\ Elisabeth.Rouy@ec-lyon.fr
}

\begin{abstract}
This article presents an approach to the shape from shading problem which is based upon the notion of viscosity solutions to the shading partial differential equation, in effect a Hamilton-Jacobi equation. The power of this approach is twofolds: 1) it allows nonsmooth, i.e. nondifferentiable, solutions which allows to recover objects with sharp troughs and creases and 2) it provides a framework for deriving a numerical scheme for computing approximations on a discrete grid of these solutions as well as for proving its correctness, i.e. the convergence of these approximations to the solution when the grid size vanishes.

Our work extends previous work in the area in three aspects. First, it deals with the case of a general illumination in a simpler and a more general way (since they assume that the solutions are continuously differentiable) than in the work of Dupuis and Oliensis [9]. Second, it allows us to prove the existence and uniqueness of "continuous" solutions to the shading equation in a more general setting (general direction of illumination) than in the work of Rouy and Tourin [24], thereby extending the applicability of shape from shading methods to more realistic scenes. Third, it allows us to produce an approximation scheme for computing approximations of the "continuous" solution on a discrete grid as well as a proof of their convergence toward that solution.
\end{abstract}

Keywords: Shape from Shading, viscosity solutions, existence and uniqueness of a solution, Hamilton-Jacobi equations, dynamic programming principle, approximation and numerical schemes.

\section{Introduction}

Shape from shading has been a central problem in the field of computer vision since the early days. The problem is to compute the three-dimensional shape of a surface from the brightness variations in a black and white image of that surface. The work in our field was pioneered by Horn who was the first to pose the problem as that of finding the solution of a nonlinear first-order partial differential equation called the brightness equation [15]. This initial idea was limited by the particular numerical method that was used (the method of characteristics) and was enriched by posing the problem as a variational problem [14] within which additional constrains such as those provided by occluding contours [17] 
can be taken into account. The book [13] contains a very nice panorama of the research in this area up to 1989. Questions about the existence and uniqueness of solutions to the problem were simply not even posed at that time with the important exception of the work of Bruss [4]. These questions as well as those related to the convergence of numerical schemes for computing the solutions became central in the last decade of the 20th century. Brightness equations that do not admit continuously differentiable solution were produced $[3,16]$, Durou and his co-workers showed that some well-known numerical schemes were in fact almost never convergent [10] and exhibited a continuous family of ambiguous solutions [11]. A breakthrough was achieved by people who realized that control theory could be brought to bear on this problem. Dupuis and Oliensis showed that this theory provided a way of constructing numerical schemes with provable convergence properties in the case where a continuously differentiable solution existed [9]. More significantly perhaps, P.-L. Lions, Rouy and Tourin used the theory of viscosity solution of Hamilton-Jacobi equations to characterize the existence and uniqueness of weak solutions to the brightness equation and to come up with provably convergent numerical schemes to compute them $[24,21]$. In doing so, they considerably generalized the applicability of shape from shading since solutions could be only continuous and they opened the way to the mathematically well-posed use of such constraints as occluding edges and shadows as well as general light sources.

In this article we revisit one of the simplest versions of the shape from shading problem, the idea being that the tools that we develop here will be extendable to more general and realistic situations. We therefore assume that the camera performs an orthographic projection of the scene (hence a simple affine camera model as opposed to a pinhole), that the scene is illuminated by a single point source at infinity, that its reflectance is Lambertian and its albedo constant and equal to 1 . We also assume that there are no shadows and no occluding boundaries and that the distance from the camera to the scene is known on the boundary of the image. Admittedly, these hypotheses may appear a bit restrictive. In fact they are not in the sense that they can be generalized without drastically changing the mathematical analysis that is done in this paper. But this will be the subject of another paper.

We denote by $u$ the distance of the points in the scene to the camera, $I$ the image intensity, $\mathbf{L}=(\alpha, \beta, \gamma)$ the unit vector representing the direction of the light source $(\gamma>0)$, and $\mathbf{l}=(\alpha, \beta)$. The image is modelled as a function from the closure $\bar{\Omega}$ of an open set $\Omega$ of $\mathbb{R}^{2}$ into the closed interval $[0,1], I: \bar{\Omega} \longrightarrow[0,1]$. Given our hypotheses, the shape from shading problem is, given $I$ and $\mathbf{L}$, to find a function $u: \bar{\Omega} \longrightarrow \mathbb{R}$ satisfying the brightness equation:

$$
\forall x \in \Omega, \quad I(x)=\frac{-\nabla u(x) \cdot 1+\gamma}{\sqrt{1+|\nabla u(x)|^{2}}}
$$

with the Dirichlet boundary conditions $\forall x \in \partial \Omega, \quad u(x)=\varphi(x)$,

$\varphi$ being continuous on $\partial \Omega$. 
Note that in the case where the light source is in the same direction as the direction of projection (it is the case considered in [24]) we have $\mathbf{L}=(0,0,1)$, and the PDE (1) is equivalent to an Eikonal equation:

$$
|\nabla u(x)|=\sqrt{\frac{1}{I(x)^{2}}-1} .
$$

Note also that (1) is a Hamilton-Jacobi equation and can be rewritten as $H(x, \nabla u(x))=0$, where $H$ is the Hamiltonian (see section 5).

The paper is organized as follows. In section 2 we briefly introduce the notion of viscosity solutions of Hamilton-Jacobi solutions. In sections 3 and 4 we give classical theorems of existence and uniqueness of a (viscosity) solution. In section 5 we apply these theorems to the shape from shading problem. In section 6 we propose a numerical scheme for solving the brightness equation and prove its convergence to the continuous solution. In section 7 we show some experimental results on synthetic and real images and conclude in section 8 .

\section{Viscosity solutions}

The notion of viscosity solutions of Hamilton-Jacobi equations has been introduced by Crandall and Lions $[6,20,8,7]$. It is a very nice way of making quantitative and operational the intuitive idea of weak solutions of first-order (and for that matter, second-order) Partial Differential Equations (PDEs). In the context of the shape from shading problem we are only concerned with first-order PDEs.

Let us consider a Hamilton-Jacobi equation, i.e.:

$$
H(x, u(x), \nabla u(x))=0 \quad \text { in } \quad \Omega, \quad \text { an open set of } \mathbb{R}^{n},
$$

where $H$ is a continuous function from $\bar{\Omega} \times \mathbb{R} \times \mathbb{R}^{n}$ to $\mathbb{R}$. We start with the definition of viscosity subsolution and supersolution:

Definition 1 A viscosity subsolution of equation (4) is a function u satisfying $\forall \phi \in C^{1}(\Omega), \forall x_{0} \in \Omega$ local maximum of $(u-\phi), \quad H\left(x_{0}, u\left(x_{0}\right), \nabla \phi\left(x_{0}\right)\right) \leq 0$.

A viscosity supersolution of equation (4) is a function $u$ satisfying

$\forall \phi \in C^{1}(\Omega), \forall x_{0} \in \Omega$ local minimum of $(u-\phi), \quad H\left(x_{0}, u\left(x_{0}\right), \nabla \phi\left(x_{0}\right)\right) \geq 0$.

Finally,

- $u \in B U C(\Omega)$ (the set of bounded uniformly continuous functions) is a continuous viscosity solution of equation (4) if it is both a subsolution and a supersolution.

- $u$ is a discontinuous viscosity solution if $u^{*}$ (respectively $u_{*}$ ) is a subsolution of $H_{*}$ (respectively a supersolution of $\left.H^{*}\right): u^{*}$ (respectively $u_{*}$ ) is the upper semicontinuous (respectively lower semicontinuous) envelope of u; Of course, in this case, we do not suppose $H$ continuous on $\bar{\Omega}$. 
As we see in the definition, viscosity solutions are not necessarily differentiable! In this sense they are weak solutions of (4). Nonetheless, the following proposition ensures the coherence between continuous viscosity and classical solutions.

Proposition 1 Let $u$ be differentiable in $\Omega$ and a classical solution of (4). If $u \in B U C(\Omega)$, then it is a viscosity solution.

Let $u$ be a viscosity solution of (4). If $u$ is differentiable in $\Omega$, then it is a classical solution.

For more details on the notion of viscosity solutions, the interested reader is referred to $[20,2]$.

In the shape from shading application we consider in this article, we are interested in a special case of equation (4) where the Hamiltonian $H$ does not depend upon the function $u$ :

$$
H(x, \nabla u(x))=0 \quad \text { in } \quad \Omega, \text { an open set of } \mathbb{R}^{2},
$$

We restrict ourselves from now on to this case and give general results of existence and uniqueness of viscosity solutions of (5).

\section{Uniqueness of a viscosity solution}

The uniqueness results depend upon the type of boundary conditions that we impose. In this article we deal with the Dirichlet condition. For the continuous case, Rouy and Tourin give the following uniqueness result for Halmiltonians $H$ which do not depend upon $u$ (see [24]). For more general conditions, see [21].

Theorem 1 Let $H: \bar{\Omega} \times \mathbb{R}^{2} \longrightarrow \mathbb{R}$ where $\Omega$ is a bounded open set of $\mathbb{R}^{2}$. If

1. $\forall x, y \in \Omega, \forall p \in \mathbb{R}^{2},|H(x, p)-H(y, p)| \leq w(|x-y|(1+|p|))$, where $w$ is a continuous nondecreasing function such that $w(0)=0$,

2. $H$ is continuous in $\bar{\Omega} \times \mathbb{R}^{2}$ and convex with respect to $\nabla u$,

3. there exists a strict viscosity subsolution $\underline{u} \in C^{1}(\Omega) \cap C(\bar{\Omega})$ of (5) (i.e. such that $H(x, \nabla \underline{u}(x))<0$ for all $x$ in $\Omega)$,

then there exists at most one continuous viscosity solution of (5) verifying $u=\varphi$ in $\partial \Omega$, where $\varphi \in C(\partial \Omega)$.

For the discontinuous case, we have a strong uniqueness result (see [2]):

Theorem 2 if $H$ satisfies the hypotheses "HNCL" (defined below) and if there exists a constant $C>0$ such that for all $p$ in $\mathbb{R}^{2}$,

- $\forall x \in \partial \Omega, H(x, p+\lambda \eta(x)) \leq 0 \quad \Longrightarrow \quad \lambda \leq C(1+|p|), \eta(x)$ is the unit outward pointing normal vector to $\partial \Omega$.

- $H(x, p-\lambda \eta(x)) \rightarrow+\infty \quad$ uniformly with respect to $x$, when $\lambda \rightarrow+\infty$;

then for all subsolution $u$ and supersolution $v$ (in discontinuous sense), $u \leq v$ in $\Omega$. 
We then have the uniqueness of discontinuous solution on $\Omega$ (but not in $\bar{\Omega}$ !). The hypotheses "HNCL" are:

1. There exists a function $m_{R}$ which goes to zero at zero, such that $\forall x, y \in \Omega, \forall p \in \mathbb{R}^{2},|H(x, p)-H(y, p)| \leq m_{R}(|x-y|(1+|p|))$

2. For all $x$ in $\Omega, H(x, p)$ is convex with respect to $p$.

3. There exists a function $\Phi$ of class $C^{1}$ in $\Omega$, continuous in $\bar{\Omega}$ such that $H(x, D \Phi(x)) \leq \delta<0$ in $\Omega$

4. There exists a function $m_{R}$ which goes to zero at zero, such that $\forall x \in \partial \Omega$, $\forall p, q \in \mathbb{R}^{2},|H(x, p)-H(x, q)| \leq m_{R}(|p-q|)$.

\section{Existence of viscosity solution, value functions and boundary compatibility conditions}

We recall here theorems of existence of viscosity solutions in the special case where the Hamiltonian $H$ appearing in equation (5) is convex with respect to $\nabla u$. We also consider the case of Dirichlet boundary conditions. In the case of continuous solutions on $\bar{\Omega}$, the existence theorem can be interpreted as giving compatibility constraints for the boundary conditions.

We note $H^{*}$ the Legendre transform of $H$ :

$$
H^{*}(x, q)=\sup _{p \in \mathbb{R}^{2}}\{p . q-H(x, p)\} \leq+\infty .
$$

The following theorem is a special case of theorem 5.3 in [20].

\section{Theorem 3 If}

1. $H \in C\left(\bar{\Omega} \times \mathbb{R}^{2}\right)$ is convex with respect to $\nabla u$ for all $x$ in $\bar{\Omega}$,

2. $H(x, p) \rightarrow+\infty$ when $|p| \rightarrow+\infty$ uniformly with respect to $x \in \bar{\Omega}$,

3. inf $f_{p \in \mathbb{R}^{2}} H(x, p) \leq 0$ in $\bar{\Omega}$,

4. $\forall x, y \in \partial \Omega, \varphi(x)-\varphi(y) \leq L(x, y)$;

( where $L(x, y)=\inf _{\xi \in C_{x, y}}\left\{\int_{0}^{T_{0}} H^{*}\left(\xi(s),-\xi^{\prime}(s)\right) d s\right.$ and $C_{x, y}=\{\xi \mid \xi(0)=$ $\left.\left.x, \xi\left(T_{0}\right)=y, \forall t \in\left[0, T_{0}\right], \xi(t) \in \bar{\Omega}, \xi^{\prime} \in L^{\infty}\left(0, T_{0}\right)\right\}\right)$

then the function $u$ defined in $\bar{\Omega}$ by:

$$
u(x)=\inf _{\xi \in C_{x, y}, y \in \partial \Omega}\left\{\int_{0}^{T_{0}} H^{*}\left(\xi(s),-\xi^{\prime}(s)\right) d s+\varphi\left(\xi\left(T_{0}\right)\right)\right\}
$$

is a continuous viscosity solution of equation (5) verifying $u=\varphi$ in $\partial \Omega$.

Remark: if the hypotheses of theorem 1 are satisfied, we can reformulate its conclusion as follows: The equation (5) has a unique viscosity solution iff

$$
\forall x, y \in \partial \Omega, \quad \varphi(x)-\varphi(y) \leq L(x, y) .
$$
of [1])

At last, for discontinuous solutions, we have the theorem (see theorem V.4.13 
Theorem 4 If $H(x, p)=\sup _{q \in Q}\{-f(x, q) \cdot p-l(x, q)\}$, with $Q \subset \mathbb{R}^{2}$ compact, $f: \mathbb{R}^{2} \times Q \mapsto \mathbb{R}^{2}$ Lipschitz continuous in $x$ uniformly in $q$, with $l: \mathbb{R}^{2} \times Q \mapsto \mathbb{R}$ continuous such that there exist constants $\omega_{l}$ and $M$ such that

$|l(x, q)-l(y, q)| \leq \omega_{l}(|x-y|)$ and $|l(x, q)| \leq M, \quad \forall x, y \in \mathbb{R}^{2}$ and $q \in Q ;$

then $u$ (defined above) is a viscosity solution of (5) with Dirichlet condition (2)

in the discontinuous sense.

One very nice thing about these theorems is that not only do they guarantee the existence of viscosity solutions, but they also show that the solution is the value function of an optimal control problem and provide a way of constructing one explicitely.

\section{Application to the shape from shading problem}

As shown in section 1 , the shape from shading equation is of the form $H(x, \nabla u)=0$, where $H(x, p)=I(x) \sqrt{1+|p|^{2}}+p \cdot \mathbf{l}-\gamma$. It is easy to verify that if $I(x)$ does not reach the value 1 within $\Omega$, i.e. $0 \leq I(x)<1 \quad \forall x \in \Omega$, the function $\tilde{u}:(x, y) \longmapsto \frac{1}{\gamma}(-\alpha x-\beta y)$ is a strict viscosity subsolution of (5). If $I$ is Lipschitz continuous, the other hypotheses of theorem 1 are also verified (as the reader will readily check) and we conclude that there exists at most one continuous viscosity solution of the PDE

$$
\left\{\begin{array}{lr}
I(x) \sqrt{1+|\nabla u(x)|^{2}}+\nabla u(x) \cdot 1-\gamma=0 & \forall x \in \Omega \\
u(x)=\varphi(x) & \forall x \in \partial \Omega
\end{array}\right.
$$

Otherwise, under these same hypotheses, theorem 2 easily applies oneself as soon as $I>|\mathbf{1}|$; thus in this case, we have also the uniqueness of discontinuous solution on $\Omega$ (but not on $\bar{\Omega}$ !). In practice, $I$ can reach the value 1 in an arbitrary compact set in $\bar{\Omega}$. This implies that there does not exist a strict viscosity subsolution and we lose uniqueness. The loss of uniqueness is completely characterized in [21] and we summarize it here. We assume that there exists a finite collection of disjoint connected compact sets $K_{i}, i=1, \cdots, n$ such that $\{I=1\}=\cup_{i=1}^{n} K_{i}$. The main points are that the viscosity solutions of (7) are constant over the $K_{i}$ 's and, when $n>1$, we only need to specify the differences of the values of $u$ in the $K_{i}$ 's. This allows us to ignore the set $\{I=1\}$ and to work in the open set $\Omega^{\prime}=\Omega-\{I=1\}$. In other words, when the set $\{I=1\}$ is not empty we consider the problem

$$
\left\{\begin{array}{lr}
I(x) \sqrt{1+|\nabla u(x)|^{2}}+\nabla u(x) \cdot 1-\gamma=0 & \forall x \in \Omega^{\prime} \\
u(x)=\varphi(x) & \forall x \in \partial \Omega^{\prime}
\end{array}\right.
$$

rather than (7). Like this, as soon as $I$ is Lipschitz continuous, we have a uniqueness result for problem (8). The solutions of (7) are then obtained from these by choosing (almost) arbitrary values for $u$ in the $K_{i}$ 's. Another possibility is to choose among all solutions one which possesses an extra property, as in the work of M. Falcone et al. $[5,12]$ where the uniqueness is obtained by choosing the maximal solution. 
Since $I$ is continuous, $H$ is continuous in $\bar{\Omega} \times \mathbb{R}^{2}$ and convex with respect to $p$; we easily obtain, taking the derivative:

$$
\inf _{p \in \mathbb{R}^{2}} H(x, p)= \begin{cases}\sqrt{I(x)^{2}-|1|^{2}}-\gamma & \text { if } I(x)^{2} \geq \alpha^{2}+\beta^{2} . \\ -\infty & \text { otherwise. }\end{cases}
$$

Since $I^{2} \leq \alpha^{2}+\beta^{2}+\gamma^{2}=1$, we have $\inf _{p \in \mathbb{R}^{2}} H(x, p) \leq 0$. Finally, since $\Omega$ is bounded hypothesis 2 in theorem 3 is satisfied iff $I>|\mathbf{1}|$ and, if the compatibility conditions (6) are satisfied on $\partial \Omega^{\prime}$, we have obtained existence and uniqueness of a continuous viscosity solution of problem (8). In the discontinuous case, the theorem 4 applied with $A=\bar{B}(0,1), f(x, q)=-1-I(x) q$ and $l(x, q)=$ $-I(x) \sqrt{1-|q|^{2}}-\gamma$, is valid as soon as $I$ is Lipschitz in $\bar{\Omega}$ (and even if $I^{2}<\alpha^{2}+\beta^{2}$ or $I=0 !)$.

Finally, in both cases,

$$
u(x)=\inf _{\xi}\left\{\int_{0}^{T_{0}} H^{*}\left(\xi(s),-\xi^{\prime}(s)\right) d s+\varphi\left(\xi\left(T_{0}\right)\right)\right\}
$$

where $\xi \in \cup_{y \in \partial \Omega} C_{x, y}$ satisfies $\forall t \in\left[0, T_{0}\right],-\xi^{\prime}(t) \in \bar{B}(1, I(\xi(t)))$,

is a solution, and we can calculate $H^{*}$ explicitly through differential calculus:

$$
H^{*}(x, q)= \begin{cases}-\sqrt{I(x)^{2}-|q-1|^{2}}+\gamma & \text { if }|q-1| \leq I(x) \\ +\infty & \text { otherwise }\end{cases}
$$

\section{Numerical approximation of the solution}

The purpose of this section is to give an algorithm for approximating the viscosity solution of (8). We also prove that our algorithm is convergent in the sense that when the spatial mesh size tends to 0 , the discrete solution tends to the viscosity solution.

\subsection{Approximation scheme}

Let $\Omega$ be the rectangular domain $] 0, X[\times] 0, Y\left[\right.$ of $\mathbb{R}^{2}$. For all mesh size $\rho=$ $(\Delta x, \Delta y)$, we want to approximate the value of the solution $u$ at the points $\left(x_{i j}\right)=\left(i \Delta x, j \Delta y\right.$ ) by $U_{i j}$ (for $i=1 . . N$ and $j=1 . . M$ where $N=\lfloor X / \Delta x\rfloor$ and $M=\lfloor Y / \Delta y\rfloor)$.

Since $u$ is a value function, the dynamic programming principle allows to write that for all $\tau \geq 0, u(x)$ is equal to the inf of

$$
\int_{0}^{T_{0} \wedge \tau} H^{*}\left(\xi(s),-\xi^{\prime}(s)\right) d s+\varphi\left(\xi\left(T_{0}\right)\right) \chi_{\left\{T_{0} \leq \tau\right\}}\left(T_{0}\right)+u(\xi(\tau)) \chi_{\left\{T_{0}>\tau\right\}}\left(T_{0}\right),
$$

where $a \wedge b$ is the smallest of $a$ and $b$. The inf is taken over the set of the $\xi \mathrm{s}$ of $\cup_{y \in \partial \Omega^{\prime}} C_{x, y}$ satisfying $\forall t \in\left[0, T_{0}\right]-\xi^{\prime}(t) \in \bar{B}(1, I(\xi(t)))$.

$\rho$ being supposed fixed, we can choose $\tau$ sufficiently small so that for all $x_{i j} \in \Omega^{\prime}$ :

$$
u(x)=\inf _{\xi}\left\{\int_{0}^{\tau} H^{*}\left(\xi(s),-\xi^{\prime}(s)\right) d s+u(\xi(\tau))\right\} .
$$


To come up with our numerical scheme we use the following approximations:

$$
\begin{gathered}
\int_{0}^{\tau} H^{*}\left(\xi(s),-\xi^{\prime}(s)\right) d s \cong H^{*}\left(\xi(0),-\xi^{\prime}(0)\right) \tau, \quad \xi(\tau) \cong \xi(0)+\xi^{\prime}(0) \tau, \\
\bar{B}((\alpha, \beta), I(\xi(t))) \cong \bar{B}(1, I(\xi(0)))
\end{gathered}
$$

Since $\xi(0)=x$, equation (11) now becomes:

$$
\sup _{\xi^{\prime}(0) \in B_{x}}\left\{\frac{u(x)-u\left(x+\tau \xi^{\prime}(0)\right)}{\tau}-H^{*}\left(x,-\xi^{\prime}(0)\right)\right\}=0 ;
$$

where $B_{x}=\bar{B}((\alpha, \beta), I(x))$.

The final step is to approximate $u$ by a piecewise affine function in a neighbourhood of $x$, see figure 1 . The goal is to obtain a relation between $x_{i j}, x_{i \pm 1, j}$

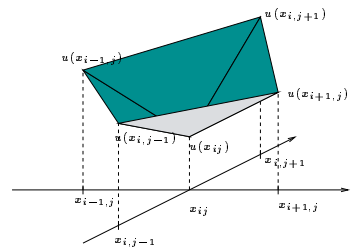

Fig. 1. Piecewise affine approximation of $u$ in a neighborhood of $x_{i j}$

and $x_{i, j \pm 1}$. In order to reach this goal, we need to compute the sup in (12) and for that matter we partition the set $Q$ in four subsets $Q_{1} \cup Q_{2} \cup Q_{3} \cup Q_{4}$,

$$
\begin{aligned}
& Q_{1}=\left\{q \in B_{x} \mid q_{x}>0, q_{y} \geq 0\right\}, Q_{2}=\left\{q \in B_{x} \mid q_{x} \leq 0, q_{y} \geq 0\right\}, \\
& Q_{3}=\left\{q \in B_{x} \mid q_{x} \leq 0, q_{y}<0\right\}, Q_{4}=\left\{q \in B_{x} \mid q_{x}>0, q_{y}<0\right\} ;
\end{aligned}
$$

over which we independently maximize (12). To simplify matters but without loss of generality, we apply a rotation of axis $O z$, and assume that $\beta=0$. In this case we note that when $x \in\{x \in \Omega|I(x) \leq|(\alpha, 0) \mid\}, Q_{1}$ and $Q_{4}$ are empty.

Let us detail the steps for $Q_{2}$. The piecewise affine approximation of $u$ yields:

$$
\frac{u\left(x_{i j}\right)-u\left(x_{i j}+\tau\left(q_{x}, q_{y}\right)\right)}{\tau} \cong-q_{x} a\left(\Delta x, x_{i j}, u_{x_{i j}}, u\right)+q_{y} d\left(\Delta y, x_{i j}, u_{x_{i j}}, u\right),
$$

where $a(\rho, x, t, u)=\frac{t-u(x-(\rho, 0))}{\rho}$ and $d(\rho, x, t, u)=\frac{t-u(x+(0, \rho))}{\rho}$. Using convexity and differential calculus we find that the maximum over $Q_{2}$ of (12) is equal to: First case: if $I(x)>\alpha$ :

$$
I(x) \sqrt{1+\left(\chi^{+}\left(a, d^{+}\right)\right)^{2}+\left(d^{+}\right)^{2}}+\alpha \chi^{+}\left(a, d^{+}\right)-\gamma
$$

where

$$
\chi^{-}(x, y)=\left\{\begin{array}{cc}
x & \text { if } x \leq-\alpha \frac{\sqrt{1+y^{2}}}{\sqrt{I^{2}-\alpha^{2}}} \\
-\alpha \frac{\sqrt{1+y^{2}}}{\sqrt{I^{2}-\alpha^{2}}} & \text { otherwise. }
\end{array}\right.
$$


and

$$
\chi^{+}(x, y)=\left\{\begin{array}{cc}
x & \text { if } x \geq-\alpha \frac{\sqrt{1+y^{2}}}{\sqrt{I^{2}-\alpha^{2}}} \\
-\alpha \frac{\sqrt{1+y^{2}}}{\sqrt{I^{2}-\alpha^{2}}} & \text { otherwise. }
\end{array}\right.
$$

Second case: if $I(x) \leq \alpha$ :

$$
I(x) \sqrt{1+a^{2}+\left(d^{+}\right)^{2}}+\alpha a-\gamma .
$$

We then collect the results of the maximization over $Q_{1}, Q_{2}, Q_{3}$ and $Q_{4}$, let $\Delta x=\Delta y=\rho$, and obtain the following numerical scheme:

$$
S(\rho, x, t, u)=0,
$$

where $S$ is equal to $\rho T(\rho, x, t, u))$, and $T$ is defined by:

1. If $x \in \Omega^{\rho}$ and $I(x)>\alpha$ then

$$
T(\rho, x, t, u)=\max \left(K_{1}(a, c, d), K_{2}(b, c, d)\right),
$$

where

$$
\begin{gathered}
K_{1}(a, c, d)=I(x) \sqrt{1+\left(\chi^{+}(a, M)\right)^{2}+M^{2}}+\alpha \chi^{+}(a, M)-\gamma, \\
K_{2}(b, c, d)=I(x) \sqrt{1+\left(\chi^{-}(-b, M)\right)^{2}+M^{2}}+\alpha \chi^{-}(-b, M)-\gamma \\
M=\max \left(c^{+}, d^{+}\right),
\end{gathered}
$$

and

$$
\begin{aligned}
a=\frac{t-u(x-(\rho, 0))}{\rho} \quad b=\frac{t-u(x+(\rho, 0))}{\rho} \quad c=\frac{t-u(x-(0, \rho))}{\rho} \\
d=\frac{t-u(x+(0, \rho))}{\rho} .
\end{aligned}
$$

2. If $x \in \Omega^{\rho}$ and $I(x) \leq \alpha$ then $T(\rho, x, t, u)=I(x) \sqrt{1+a^{2}+M^{2}}+\alpha a-\gamma$.

3. If $\mathrm{x} \in b \Omega^{\rho}$ then

$$
T(\rho, x, t, u)=t-\varphi(x) .
$$

We have noted $\Omega^{\rho}=\{x \in \Omega \mid x-(\rho, 0), x+(\rho, 0), x-(0, \rho), x+(0, \rho) \in \bar{\Omega}\}$ and $b \Omega^{\rho}=\bar{\Omega}-\Omega^{\rho}$.

\subsection{Convergence of the approximation scheme}

The following theorem is proved in [23]:

Theorem 5 Let $T(\rho, x, t, u)=0$ be an approximation scheme which can be written as $g(x, a, b, c, d)=0$, where $a, b, c, d$ are defined in (15). Let us assume that the approximation scheme $S$ satisfies the hypotheses "MSC" (defined below), then for all positive $\rho$, the scheme has a solution noted $u^{\rho}$. If furthermore $H$ satisfies the hypotheses of the strong uniqueness theorem (theorem 2), then $u^{\rho}$ converges toward the solution of the corresponding PDE when $\rho \longrightarrow 0$. 
The hypotheses "MSC" are:

1. $g$ is increasing with respect to each of the four variables $a, b, c$ and $d$;

2. there exists a bounded function $u_{0}$ such that $\forall x \in \bar{\Omega}, T\left(\rho, x, u_{0}(x), u_{0}\right) \leq 0$;

3. for all $\rho, x$ and $u, \lim _{t \rightarrow+\infty} T(\rho, x, t, u) \geq 0$;

4. $g(x, a, b, c, d) \leq 0 \Longrightarrow a, b, c$ or $d$ are bounded independently of $x$;

5. $g$ is continuous in $\bar{\Omega} \times \mathbb{R}^{4}$ and $\forall x \in \bar{\Omega}, \forall \phi \in C_{b}^{\infty}(\bar{\Omega})$,

$$
g\left(x, \partial_{x} \phi(x),-\partial_{x} \phi(x), \partial_{y} \phi(x),-\partial_{y} \phi(x)\right)=H(x, \phi(x), \nabla \phi(x))
$$

Let us emphasize the fact that when the scheme is obtained by the process described in subsection 6.1, most "MSC" hypotheses are systematically verified. In particular, it is easily to apply the theorem 5 , in the case for all $x$ in $\bar{\Omega}$, $I^{2}(x)>\alpha^{2}+\beta^{2}$. For more details, see [23].

\subsection{Algorithm for computing the solution}

Thanks to theorem 5 , we know that when $\rho=(\Delta x, \Delta y)$ tends to zero, the solutions $u^{\rho}$ of the numerical scheme (13) converge to the unique viscosity solution of equation (8). We now describe an algorithm that computes an approximation of $u^{\rho}$, for each value of $\rho>0$. It is important to keep in mind that this algorithm converges toward $u^{\rho}$ but not toward the viscosity solution. We assume that the image $I$ is known on the discrete grid $\left(x_{i j}\right)_{i=1 . . N, j=1 . . M}$. The algorithm consists of the following computation of the sequence of the values $U_{i j}^{n}, n \geq 0$ :

Algorithm 1 1. Initialisation $(n=0): U_{i j}^{0}=u_{0}\left(x_{i j}\right)$.

2. Choice of a pixel $x_{i j} \in \Omega^{\prime}$ and modification (step $\left.n+1\right)$ of $U_{i j}^{n}$ :

We choose $U^{n+1}=\sup \left\{V=\left(V_{k, l}\right)_{(k, l) \in \bar{\Omega}}\right.$ such that $\forall(k, l) \neq(i, j), V_{k l}=U_{k l}^{n}$ and $\left.g\left(\rho, x_{i j}, V_{i j}, V\right)=0\right\}$.

3. Choose the next pixel $x_{i j} \in \Omega^{\prime}$ in such a way that all pixels of $\Omega^{\prime}$ are regularly visited and go back to 2.

We have the following

Theorem 6 If the hypotheses of theorem 5 are satisfied, the algorithm 1 is welldefined (step 2 always yields a value) and the sequence $U^{n}$ is increasing and converges toward $u^{\rho}$ when $n \longrightarrow+\infty$.

Let us note

$K_{1}(t)=I(x) \sqrt{1+\left(\chi^{+}\left(D_{x}^{-} U_{i j}(t), M(t)\right)\right)^{2}+M(t)^{2}}+\alpha \chi^{+}\left(D_{x}^{-} U_{i j}(t), M(t)\right)-\gamma$,

$K_{2}(t)=I(x) \sqrt{1+\left(\chi^{-}\left(D_{x}^{+} U_{i j}(t), M(t)\right)\right)^{2}+M(t)^{2}}+\alpha \chi^{-}\left(D_{x}^{+} U_{i j}(t), M(t)\right)-\gamma$,

and

$$
K(t)=\operatorname{Max}\left(K_{1}(t), K_{2}(t)\right)
$$

where

$$
\begin{aligned}
& D_{x}^{-} U_{i j}(t)=\frac{t-U_{i-1 j}}{\Delta-\Delta_{x}^{x}} D_{x}^{+} U_{i j}(t)=\frac{U_{i+1 j}-t}{\Delta x} \\
& D_{y}^{-} U_{i j}(t)=\frac{t-U_{i j-1}}{\Delta y} D_{y}^{+} U_{i j}(t)=\frac{U_{i j+1}-t}{\Delta y}
\end{aligned}
$$




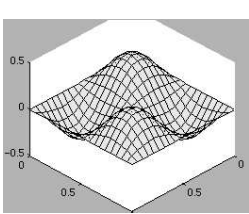

a)

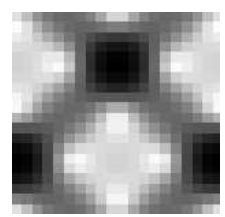

b)

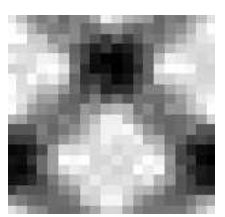

c)

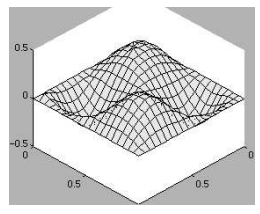

d)

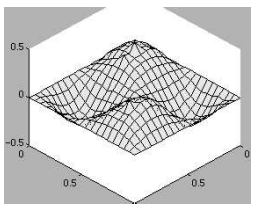

e)

Fig. 2. Results for a synthetic image generated by a sinusoidal surface sampled on a grid of size $20 \times 20$ with $\theta=28^{\circ}$ : a) original surface, b) original image, c) noisy image; d) surface reconstructed from b): $n=40, \epsilon_{1}=10.0 \%, \epsilon_{2}=9.9 \% \epsilon_{3}=15.2 \%$; e) surface reconstructed from c): $n=62, \epsilon_{1}=12.8 \%, \epsilon_{2}=10.5 \% \epsilon_{\infty}=15.7 \%$

$$
M(t)=\max \left(\left(D_{y}^{-} U_{i j}(t)\right)^{+},\left(D_{y}^{+} U_{i j}(t)\right)^{-}\right) .
$$

We note $U_{\operatorname{minj}}=\min \left(U_{i, j+1}, U_{i, j-1}\right)$.

For example, in the case where $I\left(x_{i j}\right)>\alpha$, Step 2 of algorithm 1 computes the value of $t$ such that $K(t)=0$, i.e. such that:

$$
(\underbrace{K_{1}(t)=0 \text { and } K_{2}(t) \leq 0}_{\text {Case } 1}) \quad \text { or } \quad(\underbrace{K_{2}(t)=0 \text { and } K_{1}(t) \leq 0}_{\text {Case } 2})
$$

$t$ is therefore either a root of $K_{1}$, or a root of $K_{2}$. Let us detail the procedure for finding the roots of $K_{1}$ :

1. Search for a root $t \geq U_{m i n j}$

- If $t$ is such that $D_{x}^{-} U_{i j}(t) \geq-\alpha \frac{\sqrt{1+M(t)^{2}}}{\sqrt{I^{2}-\alpha^{2}}}$ then $t$ is a root of

$$
I(x) \sqrt{1+D_{x}^{-} U_{i j}(t)^{2}+M(t)^{2}}+\alpha D_{x}^{-} U_{i j}(t)-\gamma=0 .
$$

- If $t$ is such that $D_{x}^{-} U_{i j}(t) \leq-\alpha \frac{\sqrt{1+M(t)^{2}}}{\sqrt{I^{2}-\alpha^{2}}}$ then $t$ is a root of

$$
I(x) \sqrt{1+\alpha^{2} \frac{1+M(t)^{2}}{I^{2}-\alpha^{2}}+M(t)^{2}}-\alpha^{2} \sqrt{\frac{1+M(t)^{2}}{I^{2}-\alpha^{2}}}-\gamma=0 .
$$

2. Search for a root $t \leq U_{m i n j} .(M(t)=0$ !) In that case $t$ is such that $D_{x}^{-} U_{i j}(t) \geq-\frac{\alpha}{\sqrt{I^{2}-\alpha^{2}}}$ and therefore it is the root of

$$
I(x) \sqrt{1+D_{x}^{-} U_{i j}(t)^{2}}+\alpha D_{x}^{-} U_{i j}(t)-\gamma=0 .
$$

Once we have found the roots $t_{i}, i=1,2$ de $K_{1}$ we have to verify that $K_{2}\left(t_{i}\right) \leq 0$, otherwise we must find the roots $u_{i}, i=1,2$ of $K_{2}$ such that $K_{1}\left(u_{j}\right) \leq 0$. 


\section{Experimental results}

We have tested our algorithm with synthetic images generated by shapes with several levels of regularity e.g. $C^{\infty}$ (a sinusoid, see figures 2 and 3 ), or $C^{0}$ (a pyramid, see figures 4 and 5), to demonstrate the ability of our method to deal with smooth and nonsmooth objects. We also have tested it with real images, an example is shown in figure 6 . In all results, the parameters are $n$, the number

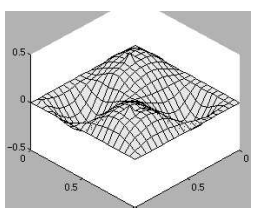

a)

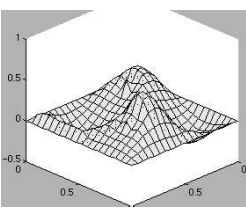

b)

Fig. 3. Sinusoidal surface of figure 2 reconstructed with an error on the parameter $\mathbf{L}$ : a) $\theta_{p}=33^{\circ}, \epsilon_{\theta}=5^{\circ}, n=37, \epsilon_{1}=11.4 \%, \epsilon_{2}=7.6 \% \epsilon_{\infty}=17.9 \%$; b) $\theta_{p}=18^{\circ}$, $\epsilon_{\theta}=10^{\circ}, n=45, \epsilon_{1}=18.3 \%, \epsilon_{2}=13.8 \%, \epsilon_{3}=41.9 \%$.

of iterations, $\epsilon_{1}, \epsilon_{2}$ and $\epsilon_{\infty}$ the relative errors of the computed surface measured according to the $L_{1}, L_{2}$ and $L_{\infty}$ norms, respectively, $\theta$ the angle of the direction of illumination with the $z$-axis.

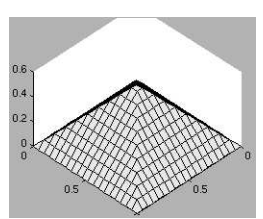

a)

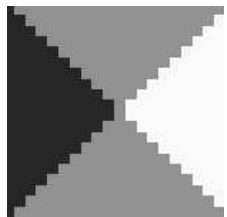

b)

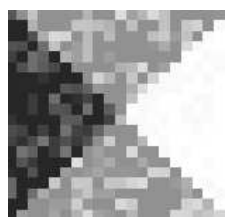

c)

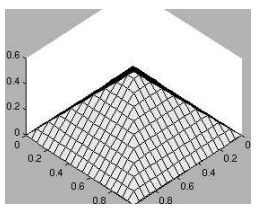

d)

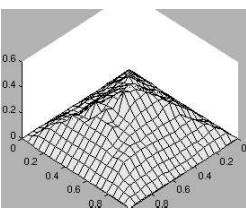

e)

Fig. 4. Results for a synthetic image generated by a pyramidal surface sampled on a grid of size $20 \times 20$ with $\theta=36^{\circ}$ : a) original surface, b) original image, c) noisy image; d) surface reconstructed from b): $n=89, \epsilon_{1}=0.4 \%, \epsilon_{2}=0.4 \% \epsilon_{\infty}=0.8 \%$; e) surface reconstructed from c): $n=91, \epsilon_{1}=23.7 \%, \epsilon_{2}=23.2 \% \epsilon_{\infty}=26.3 \%$.

In all synthetic cases we show the original object, the input image and the reconstructed surface. We then demonstrate the stability of our method with respect to two types of errors. The first type is image intensity errors due to noise. Uniformly distributed noise has been added to some pixels of the input images and the corresponding reconstructed surfaces are shown (figures 2 and 4). The second type of error is due to an incorrect estimation of the direction of 


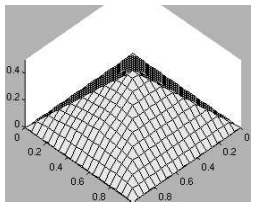

a)

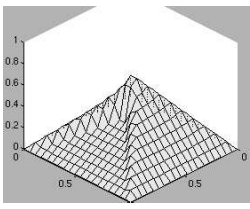

b)

Fig. 5. Pyramidal surface of figure 4 reconstructed with an error on the parameter $\mathbf{L}$ : a) $\theta_{p}=41^{\circ}, \epsilon_{\theta}=5^{\circ}, n=80, \epsilon_{1}=16.5 \%, \epsilon_{2}=15.2 \% \epsilon_{3}=19.8 \%$; b) $\theta_{p}=26^{\circ}$, $\epsilon_{\theta}=10^{\circ}, \epsilon_{1}=25.1 \%, \epsilon_{2}=14.0 \% \epsilon_{3}=28.3 \%$.

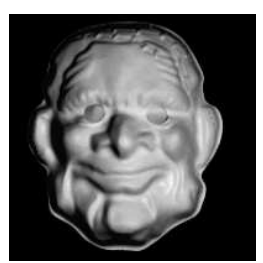

a)

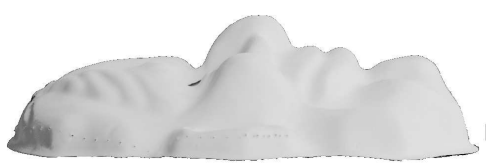

b)
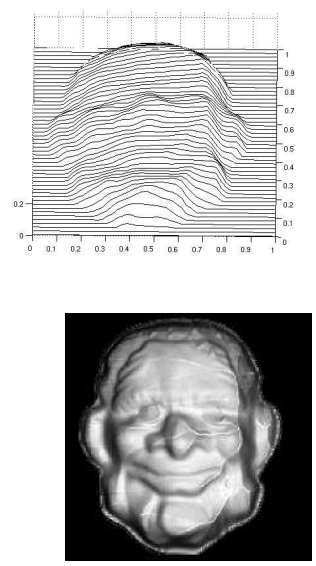

d)

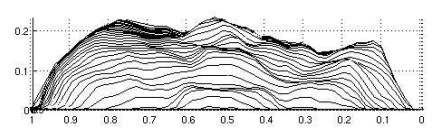

c)

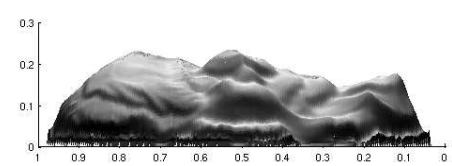

e)
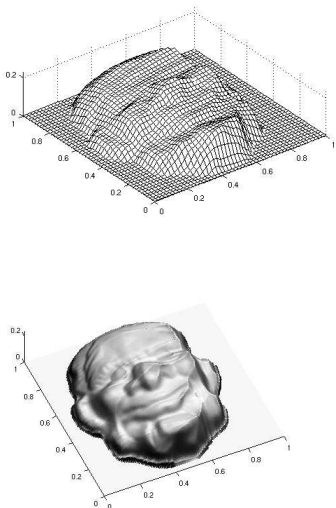

f)

Fig. 6. Experimental results with a real image: a) Original image of size 200x200, b) Lateral view (image) of the real object, c) Three views of the surface reconstructed from a), d) Synthetic image generated from the surface c), e) and f) reconstructed surface, textured with image d). 
illumination $\mathbf{L}$ (figures 3 and 5 ). We start with a smooth sinusoidal object, see figure 2 . We introduce an error $5^{\circ}$ on the parameter $\mathbf{L}$, see figure 3 . We note $\theta_{p}$ the angle used for computation; $\epsilon_{\theta}=\left|\theta-\theta_{p}\right|$.

As seen from these figures, our algorithm seems to be quite robust, not only to intensity noise (see figures 2.e and 4.e), as in [24], but also to inaccuracies in the estimation of the direction of the light source $\mathbf{L}$ (see figures 3 and 5). The pyramid example shows the remarkable ability of the numerical scheme to deal with functions which are only continuous. This example also shows the convergence of our algorithm with discontinuous images: through the recent works of Ostrov $[22,18]$, we hope to extend shortly our theory for discontinuous images.

The real image shown in figure 6.a and 6.b is a photograph of a Halloween mask taken with a standard camera with $70 \mathrm{~mm}$ focal length. The light source was far from being pointlike and at infinity and ambiant lighting due to reflections on the walls was present. The reflectance of the mask was not quite Lambertian (some highlights were visible). Unlike the case of the synthetic examples where the critical points $x$ such that $I(x)=1$ were included in the boundary conditions (i.e. their distances were supposed to be known), these distances must in this case be computed by the algorithm, making the problem ill-posed. Despite this difficulty, the results shown in figure 6.c-f are of good quality.

\section{Conclusion}

We have proposed a rigorous mathematical analysis of the simplest version of the shape from shading problem in the case of a Lambertian object illuminated by a point source at infinity and imaged by an orthographic camera. We have given hypotheses for the existence and uniqueness of a (continuous) viscosity solution, provided an approximation scheme of this solution, an algorithm for computing the solution. Unlike in [19], we have proved the convergence of our numerical scheme. Our implementation of the algorithm described in [19] shows that it fails as soon as the direction of the source of lighting is a bit too far from being parallel to the $z$-axis, the original case studied in [24].

We are extending our analysis and algorithm to more general cases.

\section{References}

1. M. Bardi and I. Capuzzo-Dolcetta. Optimal control and viscosity solutions of Hamilton-Jacobi-Bellman equations. Birkhauser, 1997.

2. G. Barles. Solutions de Viscosité des Equations de Hamilton-Jacobi. SpringerVerlag, 1994.

3. M.J. Brooks, W. Chojnacki, and R. Kozera. Shading without shape. Quarterly of Applied Mathematics, L(1):27-38, 1992.

4. A.R. Bruss. The eikonal equation: Some results applicable to computer vision. Journal of Mathematical Physics, 23(5):890-896, May 1982.

5. F. Camilli and M. Falcone. An approximation scheme for the maximal solution of the shape-from-shading model. International Conference on Image Processing, pages $49-52,1996$. 
6. M.G. Crandall. Viscosity solutions of Hamilton-Jacobi equations. In Nonlinear Problems: Present and Future, Proc. 1st Los Alamos Conf., 1981, volume 61, pages 117-125. North-Holland Math. Stud., 1982.

7. M.G. Crandall, H. Ishii, and P.L. Lions. User's guide to viscosity solutions of second order partial differential equations. Bull. Amer. Soc., 27:1-67, 1992.

8. M.G. Crandall and P.L. Lions. Viscosity solutions of Hamilton-Jacobi equations. Trans. AMS, 277:1-43, 1983.

9. Paul Dupuis and John Oliensis. An optimal control formulation and related numerical methods for a problem in shape reconstruction. The Annals of Applied Probability, 4(2):287-346, 1994.

10. J.-D. Durou and H. Maitre. On convergence in the methods of strat and smith for shape from shading. The International Journal of Computer Vision, 17(3):273-289, 1996.

11. J.-D. Durou and D. Piau. Ambiguous shape from shading with critical points. Journal of Mathematical Imaging and Vision, 12:99-108, 2000.

12. M. Falcone and M. Sagona. An algorithm for the global solution of the shapefrom-shading model. International Conference on Image Analysis and Processing, 1:596-603, 1997. LNCS 1310.

13. Berthold K. Horn and Michael J. Brooks, editors. Shape from Shading. The MIT Press, 1989.

14. Berthold K.P. Horn. Height and Gradient from Shading. The International Journal of Computer Vision, 5(1):37-75, August 1990.

15. B.K.P. Horn. Obtaining shape from shading information. In P.H. Winston, editor, The Psychology of Computer Vision. McGraw-Hill, New York, 1975.

16. B.K.P Horn, R.S. Szeliski, and A.L. Yuille. Impossible shaded images. IEEE Transactions on Pattern Analysis and Machine Intelligence, 15(2):166-169, 1993.

17. K. Ikeuchi and B.K.P Horn. Numerical shape from shading and occluding boundaries. Artificial Intelligence Journal, 17:181-184, 1981.

18. J. Kain and D.N. Ostrov. Numerical shape-from-shading for discontinuous photographic images. The International Journal of Computer Vision, 44(3):163-173, 2001.

19. R. Kimmel and J.A. Sethian. Optimal algorithm for shape from shading and path planning. Journal of Mathematical Imaging and Vision, 14(2):237-244, May 2001.

20. P.L. Lions. Generalized Solutions of Hamilton-Jacobi Equations. Number 69 in Research Notes in Mathematics. Pitman Advanced Publishing Program., 1982.

21. P.L. Lions, E. Rouy, and A. Tourin. Shape-from-shading, viscosity solutions and edges. Numer. Math., 64:323-353, 1993.

22. D.N. Ostrov. Extending viscosity solutions to eikonal equations with discontinuous spatial dependence. Nonlinear Anal., 42(4):709-736, 2000.

23. E. Prados, O. Faugeras, and E. Rouy. Shape from shading and viscosity solutions. Technical report, INRIA, 2002.

24. E. Rouy and A. Tourin. A Viscosity Solutions Approach to Shape-from-Shading. SIAM Journal of Numerical Analysis, 29(3):867-884, June 1992. 